\title{
Definition and Diagnostic Criteria for Sarcopenic Obesity: ESPEN and EASO Consensus Statement
}

\author{
Lorenzo M. Donini $^{\mathrm{a}}$ Luca Busetto $^{\mathrm{b}}$ Stephan C. Bischoff ${ }^{\mathrm{c}}$ Tommy Cederholm ${ }^{\mathrm{d}}$ \\ Maria D. Ballesteros-Pomar ${ }^{\mathrm{e}} \quad$ John A. Batsis ${ }^{f} \quad$ Juergen M. Bauer ${ }^{g} \quad$ Yves Boirie $^{\text {h }}$ \\ Alfonso J. Cruz-Jentoft ${ }^{i}$ Dror Dicker ${ }^{j}$ Stefano Frarak ${ }^{k}$ Gema Frühbeck' Laurence Genton ${ }^{m}$ \\ Yftach Gepner $^{n} \quad$ Andrea Giustinak $^{k}$ Maria Cristina Gonzalez ${ }^{\circ}$ Ho-Seong Han ${ }^{p}$ \\ Steven B. Heymsfield ${ }^{q}$ Takashi Higashiguchi ${ }^{r}$ Alessandro Laviano ${ }^{a}$ Andrea Lenzi ${ }^{\mathrm{a}}$ \\ Ibolya Nyulasis $^{s}$ Edda Parrinello ${ }^{a}$ Eleonora Poggiogalle ${ }^{a}$ Carla M. Prado $^{t}$ Javier Salvador ${ }^{\mathrm{u}}$ \\ Yves Rolland $^{\mathrm{v}}$ Ferruccio Santini $^{\mathrm{w}}$ Mireille J. Serlie ${ }^{\mathrm{X}}$ Hanping Shi ${ }^{\mathrm{y}}$ Cornel C. Sieber ${ }^{\mathrm{z}}$ \\ Mario Siervo $^{A}$ Roberto Vettor ${ }^{b}$ Dennis T. Villareal ${ }^{B}$ Dorothee Volkert ${ }^{z}$ Jianchun $\mathrm{Yu}^{\mathrm{C}}$ \\ Mauro Zamboni ${ }^{\mathrm{D}}$ Rocco Barazzoni ${ }^{\mathrm{E}}$

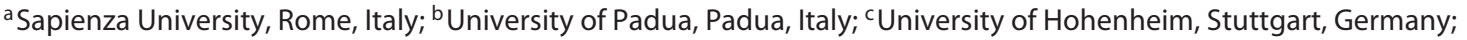

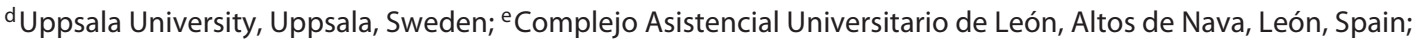 \\ fUniversity of North Carolina at Chapel Hill, Chapel Hill, NC, USA; ${ }^{9}$ University of Heidelberg, Heidelberg, Germany; \\ hUniversity of Clermont Auvergne, INRA, CRNH, CHU Clermont-Ferrand, Clermont-Ferrand, France; ' $\mathrm{Hospital}$ \\ Universitario Ramón y Cajal (IRYCIS), Madrid, Spain; 'Sackler Faculty of Medicine, Tel-Aviv University, Tel-Aviv, Israel; \\ kSan Raffaele, Vita-Salute University and IRCCS Hospital, Milan, Italy; 'Clínica Universidad de Navarra, CIBEROBN, \\ IdiSNA, Pamplona, Spain; ${ }^{\mathrm{m} H o ̂ p i t a u x}$ Universitaires de Genève, Genève, Switzerland; ${ }^{\mathrm{T}}$ Tel-Aviv University, Tel-

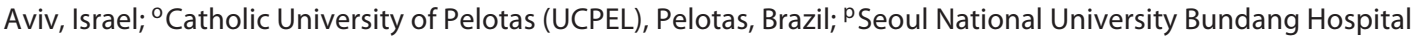 \\ (SNUBH), Seoul, South Korea; ${ }^{a}$ Pennington Biomedical Research Center, Baton Rouge, LA, USA; ${ }^{r}$ Fujita Health \\ University School of Medicine, Aichi, Japan; ${ }^{5}$ Monash University, Clayton, VIC, Australia; ${ }^{t}$ University of Alberta, \\ Edmonton, AB, Canada; "Universidad de Navarra, CIBEROBN, IdiSNA, Pamplona, Spain; ${ }^{\vee}$ Gerontopole of Toulouse,

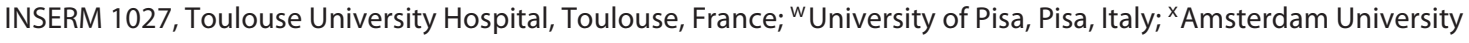 \\ Medical Centers, Amsterdam, The Netherlands; ${ }^{y}$ Beijing Shijitan Hospital, Capital Medical University, Beijing, \\ China; ${ }^{\mathrm{F}}$ Friedrich-Alexander-Universität Erlangen-Nürnberg, Nuremberg, Germany; ${ }^{\mathrm{A}}$ University of Nottingham, \\ Nottingham, UK; ${ }^{B}$ Baylor College of Medicine, Houston, TX, USA; ${ }^{C}$ Peking Union Medical College Hospital, Beijing, \\ China; ${ }^{D}$ University of Verona, Verona, Italy; ${ }^{\mathrm{E}}$ Department of Medical, Surgical and Health Sciences, University of \\ Trieste, Trieste, Italy
}

\section{Keywords}

Obesity · Sarcopenia $\cdot$ Sarcopenic obesity

Joint consensus statement of the European Society for Clinical Nutrition and Metabolism (ESPEN) and the European Association for the Study of Obesity (EASO). The consensus statement has been simultaneously published in Clinical Nutrition (DOI 10.1016/j. clnu.2021.11.014).

karger@karger.com www.karger.com/ofa

Karger $\stackrel{\text { ' }}{5}$

GOPEN ACCESS (c) 2022 The Author(s). Published by Elsevier Ltd. on behalf of European Society for Clinical Nutrition and Metabolism and Obesity Facts published by S. Karger AG. This article is published under the Creative Commons CC-BY license.

This article is licensed under the Creative Commons Attribution 4.0 International License (CC BY) (http://www.karger.com/Services/ OpenAccessLicense). Usage, derivative works and distribution are permitted provided that proper credit is given to the author and the original publisher.

\section{Abstract}

Introduction: Loss of skeletal muscle mass and function (sarcopenia) is common in individuals with obesity due to metabolic changes associated with a sedentary lifestyle, adipose tissue derangements, comorbidities (acute and chronic diseases) and during the ageing process. Co-existence of excess adiposity and low muscle mass/function is referred to as sarcopenic obesity (SO), a condition increasingly recognized for
Correspondence to:

Lorenzo M. Donini, lorenzomaria.donini@uniromal.it

Rocco Barazzoni, barazzon@units.it 
its clinical and functional features that negatively influence important patient-centred outcomes. Effective prevention and treatment strategies for SO are urgently needed, but efforts are hampered by the lack of a universally established SO definition and diagnostic criteria. Resulting inconsistencies in the literature also negatively affect the ability to define prevalence as well as clinical relevance of SO for negative health outcomes. Aims and Methods: The European Society for Clinical Nutrition and Metabolism (ESPEN) and the European Association for the Study of Obesity (EASO) launched an initiative to reach expert consensus on a definition and diagnostic criteria for SO. The jointly appointed international expert panel proposes that $\mathrm{SO}$ is defined as the co-existence of excess adiposity and low muscle mass/function. The diagnosis of SO should be considered in at-risk individuals who screen positive for a co-occurring elevated body mass index or waist circumference, and markers of low skeletal muscle mass and function (risk factors, clinical symptoms, or validated questionnaires). Diagnostic procedures should initially include assessment of skeletal muscle function, followed by assessment of body composition where presence of excess adiposity and low skeletal muscle mass or related body compartments confirm the diagnosis of SO. Individuals with $\mathrm{SO}$ should be further stratified into stage I in the absence of clinical complications or stage II if cases are associated with complications linked to altered body composition or skeletal muscle dysfunction. Conclusions: ESPEN and EASO, as well as the expert international panel, advocate that the proposed SO definition and diagnostic criteria be implemented into routine clinical practice. The panel also encourages prospective studies in addition to secondary analysis of existing data sets, to study the predictive value, treatment efficacy and clinical impact of this SO definition.

(C) 2021 The Author(s)

Published by S. Karger AG, Basel

\section{Introduction}

Sarcopenic obesity (SO) is a clinical and functional condition characterized by the coexistence of obesity, characterized by excess fat mass (FM), and sarcopenia [1, 2]. Sarcopenia, defined as low skeletal muscle mass and function, has been identified and described as a geriatric syndrome with a multifactorial aetiology whose prevalence increases with age [3-5].

Loss of skeletal muscle mass and function generally occurs with ageing and is commonly paralleled by relative or absolute body fat gain; this process favours the potential development and onset of SO. However, sarcopenia may arise in individuals with obesity at any age. Obesity can independently lead to loss of muscle mass and function, due to the negative impact of adipose tissue-dependent metabolic derangements, such as oxidative stress, inflammation and insulin resistance, all of which negatively affect muscle mass [6]. Additionally, individuals with obesity have a high prevalence of chronic non-communicable diseases that negatively impact muscle metabolism (both anabolism and catabolism). Sedentary lifestyle may also play a relevant role, being both a primary cause and a consequence of both sarcopenia and obesity, which may be exacerbated by comorbidities. Importantly, therapeutic weight loss targeting excess fat inevitably leads to loss of variable amounts of skeletal muscle mass, which may be more pronounced in individuals with predisposing catabolic conditions (chronic diseases, ageing), following bariatric surgery (particularly in the absence of nutritional supervision and follow-up) or in the presence of long-lasting inappropriate or unbalanced dietary regimens (specifically low protein intake) and weight cycling [7-11].

On the other hand, sarcopenia may directly facilitate fat accumulation through reduced total energy expenditure, and obesity and sarcopenia may therefore synergistically enhance one another with vicious cycling of fat gains and muscle loss through reduced mobility, dependency and disability. From a clinical standpoint, SO potentially leads to a cumulative risk derived from the two individual clinical situations [12-15]. Moreover, the cross-talk between adipose tissue, skeletal muscle and bone supports the evidence of an increasingly complex picture in which the pathophysiological and clinical alterations of bone structure and osteoporosis may also play a role [16].

The negative clinical consequences of SO are of paramount importance. Sarcopenic obesity has been so far identified through various definitions and diagnostic constructs, but it has been consistently demonstrated to be a strong and independent risk factor for frailty, comorbidities and mortality in various highly prevalent disease conditions, as well as for mortality in the general and especially in the older population [17-19].

However, the lack of universally recognized diagnostic criteria for SO clearly affects patient identification, reliable assessment of SO prevalence as well as the assessment of SO-related outcomes, its cost and, consequently, public health policy. Most importantly, the lack of diagnostic criteria has a strong negative impact on the development of SO prevention and treatment strategies. Building a consensus on the definition and the diagnostic cri- 
teria of SO is therefore an urgent, unmet clinical priority. The European Society for Clinical Nutrition and Metabolism (ESPEN) and the European Association for the Study of Obesity (EASO) have thus promoted and developed an SO initiative involving an international expert panel. The panel started its activity by performing a systematic review aimed at analysing the available scientific literature on definitions and diagnostic criteria for SO which have been applied in human studies [20]. Its results confirmed a marked heterogeneity in definitions and diagnostic approaches, due to different definitions of obesity and sarcopenia, differences in methodologies to assess body composition and function, as well as in the applied reference values for the variables that have been used (different cut-offs and divergent statistical stratification methods).

Based on these results, a procedure was promoted to reach consensus on: (1) definition of SO; (2) diagnostic procedures - including approach to screening, diagnostic and staging criteria, at the level of both potential gold standards and acceptable surrogates with wide clinical applicability; (3) methodologies to be employed and related cut-off values.

\section{Methods}

The consensus initiative followed standard operating procedures for ESPEN guidelines and consensus papers [21]. A pool of 38 international researchers (all authors of the present study) from different areas (experts in obesity or sarcopenia, nutritionists, dietitians, geriatricians, experts in body composition) based in 16 countries on four continents contributed to the consensus process. Five members of the group (L.M.D., L.B., S.B., T.C. and R.B.) coordinated the study activities.

The consensus procedure used a four-phase Delphi process consisting of sequential rounds of web-based questionnaires. The first round took place in the first months of 2020 after finalization of the research questions by the steering committee. All the following phases of the consensus procedure (2nd round: July 2020; 3rd round: December 2020; 4th round: February 2021) were based on the feedback from the previous online voting used to reduce the number of items and to modify the previous research questions in order to reach the highest degree of acceptance at the following online voting.

During all the phases, participants were asked to rank each of the statements individually on a 5-point Likert scale (strongly disagree; disagree; neither agree nor disagree; agree; strongly agree) justifying their choice. Statements that met predetermined consensus thresholds (acceptance: $>75 \%$ of respondents providing a positive result - agree or strongly agree - on the Likert scale) were brought forward to the next consensus step.

\section{Results}

After four sequential rounds of web-based questionnaires, a very strong approval basis [agreement (agree + strongly agree $)=93.6 \%$ ] was reached for a global proposal encompassing SO definition, screening, diagnosis and staging with a decision algorithm to guide the patient identification and diagnostic procedure.

\section{Definition of Sarcopenic Obesity}

Sarcopenic obesity is defined as the co-existence of obesity and sarcopenia.

\section{Diagnostic Procedures}

The evaluation of individuals with suspected SO will be structured on two different levels: screening and diagnosis. The latter will allow a staging of the disease on two levels (Fig. 1).

\section{a. Screening}

Screening for SO is based on the concomitant presence of an elevated body mass index (BMI) or waist circumference with ethnicity-specific cut points [22-30] and surrogate indicators of sarcopenia (e.g., clinical symptoms, risk factors; Table 1) or validated questionnaires (e.g., SARC-F in older subjects) [31, 32]. The panel suggests to adopt the cut points given by the WHO for BMI $[22,23]$ and the references given by the National Institutes of Health [28] and Misra et al. [29] for waist circumference, respectively, for Caucasian and Asian populations. In online supplementary Table S1 (for all online suppl. material, see www.karger.com/doi/10.1159/000521241) are given other reference cut points to be considered in specific situations [22-30]. Future research should aim at defining the best cut points to be considered in research and clinical practice concerning SO.

\section{b. Diagnosis}

Diagnosis to confirm or reject SO should always follow a positive screening result. Both altered skeletal muscle functional parameters and altered body composition are needed to establish a firm diagnosis of SO. The diagnostic evaluation will primarily be performed when techniques and devices for body composition measurements are available.

The diagnosis of SO will be performed in two steps by sequentially assessing:

1. Skeletal muscle functional parameters: the group supports the assessment of skeletal muscle strength [e.g., hand-grip strength (HGS), knee extensor strength (ad- 


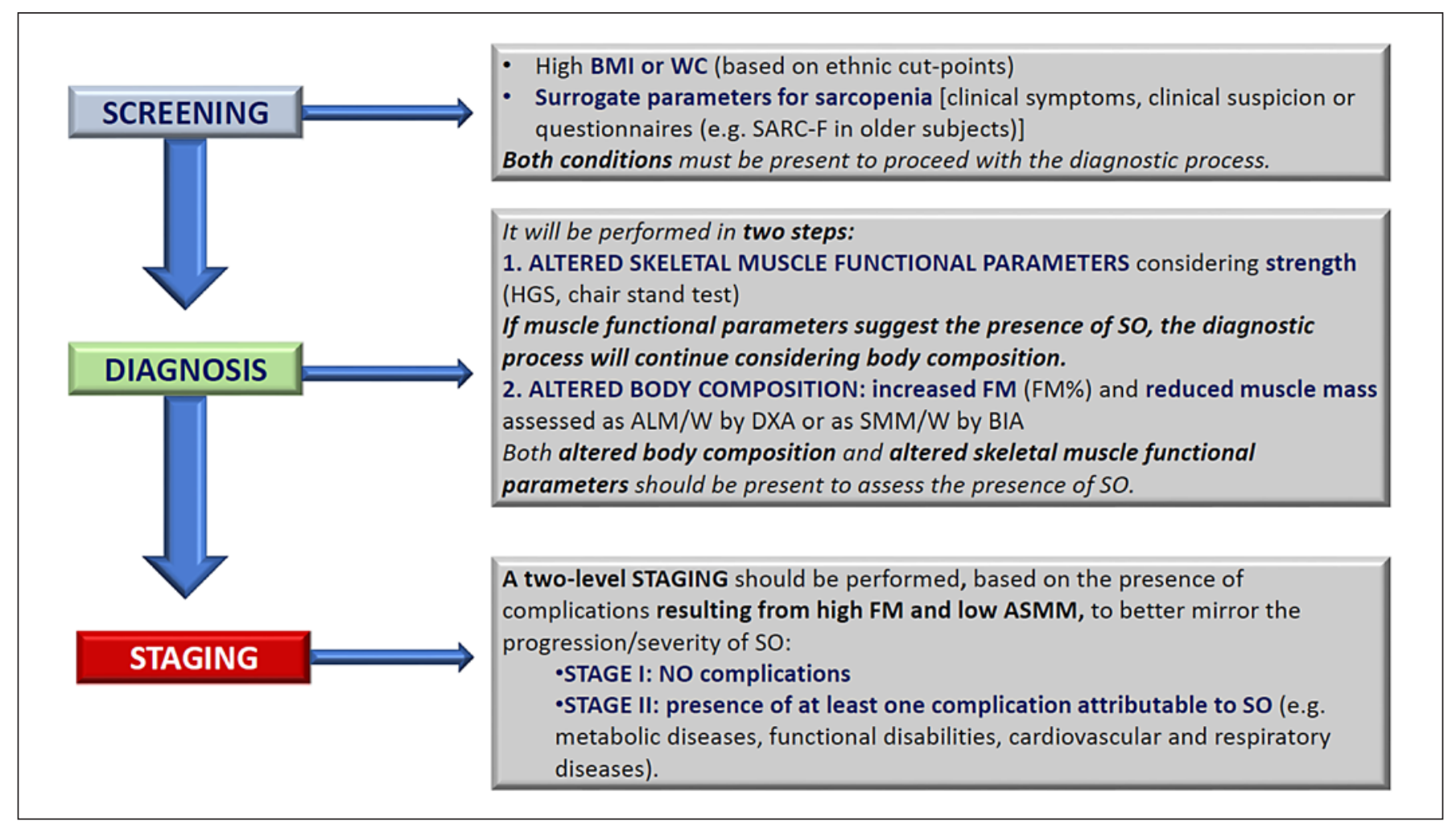

Fig. 1. Diagnostic procedure for the assessment of sarcopenic obesity. ALM/W, appendicular lean mass adjusted to body weight; ASMM, absolute skeletal muscle mass; BIA, bioelectrical impedance analysis; BMI, body mass index; DXA, dual X-ray absorptiometry; FM, fat mass; HGS, handgrip strength; SMM/W, total skeletal muscle mass adjusted by weight; SO, sarcopenic obesity; WC, waist circumference; SARC-F, strength, assistance with walking, rising from a chair, climbing stairs and falls.

justed for body mass in populations where data are available) or chair-stand test (5-time sit-to-stand test; 30 -s chair stand test)]. With regard to muscle functional parameters, cut-off points need to be validated as reference values for sex, ethnicity and age stratum [33-75]. Moreover, as discussed below, various studies suggest the necessity to adjust HGS to body mass [76, 77]. If low skeletal muscle functional parameters are detected, the diagnostic algorithm will continue with the assessment of body composition.

2. Body composition: the group supports its assessment by dual-energy X-ray absorptiometry (DXA), or bioelectrical impedance analysis (BIA) as an alternative second choice. Computerized tomography (CT) should be used when possible (e.g., in patients undergoing CT scans for additional diagnostic reasons as commonly occurring in oncology).

The panel suggests to adopt the cut points given by Dodds et al. [38] and Chen et al. [43] for HGS (for Cau- casian and Asian populations, respectively), the references given by Gallagher et al. [50] for FM, by Janssen et al. [61] for skeletal muscle mass adjusted by weight (SMM/W) and by Batsis et al. [65] for appendicular lean mass adjusted to body weight (ALM/W). In online supplementary Table S2 are given other reference cut points to be considered in specific situations [38-75]. Future research should aim at defining the best cut points to be considered in research and clinical practice concerning SO.

\section{c. Staging}

When the SO diagnosis is established, a two-level staging should be performed, which is based on the presence of complications and which aims to stratify patients based on the severity of SO. SO stages should be defined as follows:

Stage I: no complications attributable to altered body composition and skeletal muscle functional parameters 
Table 1. Clinical symptoms or suspicion factors for the screening of sarcopenic obesity

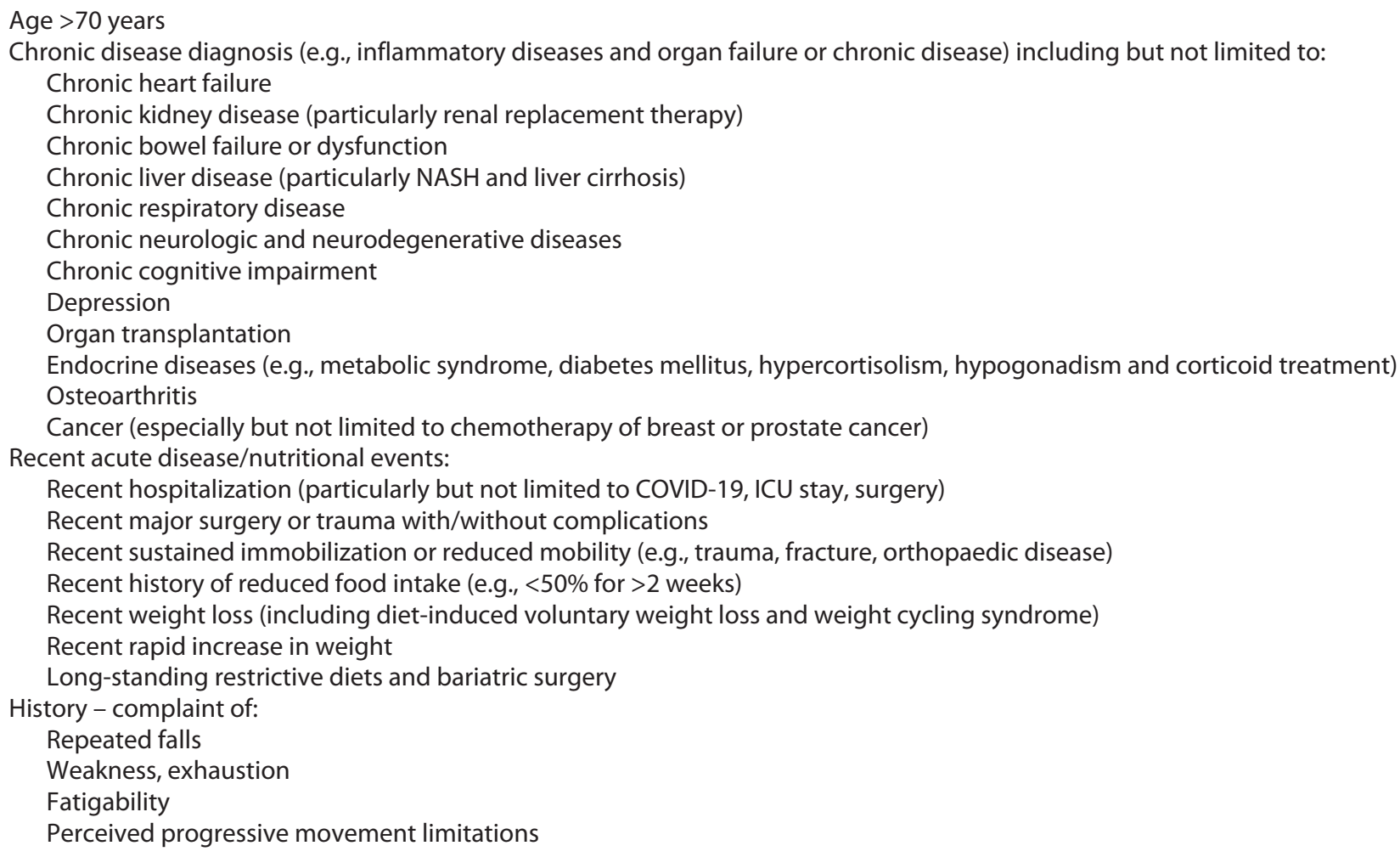

Stage II: Presence of at least one complication attributable to altered body composition and skeletal muscle functional parameters (e.g., metabolic diseases, disabilities resulting from high FM and/or low muscle mass, cardiovascular and respiratory diseases)

\section{Discussion}

Our process provided a consensus definition of SO and importantly, a structured, two-level algorithm (screening and diagnosis), in evaluating patients who may have SO. The latter will be followed by SO staging.

\section{Definition of Sarcopenic Obesity}

Sarcopenic obesity is characterized by the combination of obesity, defined by high body fat percentage, AND sarcopenia, defined as low skeletal muscle mass accompanied by low muscle function. SO needs to be considered as a unique clinical condition, different from obesity or sarcopenia alone. This is due to the existence of: (1) bidirectional, pathogenic interaction between body FM accumulation and loss of skeletal muscle mass and func-

Definition and Diagnostic Criteria for Sarcopenic Obesity tion; (2) negative clinical interactions between obesity and sarcopenia, leading to a synergistically higher risk for metabolic disease and functional impairment compared to those caused by the cumulative risk from each separate condition $[78,79]$.

The group supports that sarcopenia and obesity be identified as distinct phenotypic traits. Despite pathophysiological interactions that lead to vicious cycles with potential mutual synergistic worsening of both conditions, there are currently insufficient clinical data to suggest and support an integrated index for SO definition that simultaneously takes into account body fat and muscle mass. In a recent systematic review, we found that unified parameters with both fat and muscle measurements related in a single criterion were used for SO definition in only 6 out of 75 selected studies. Moreover, the unified parameters differed in the respective studies [20].

The group however supports that current definitions of obesity and sarcopenia should not be automatically applied to define SO. In particular, sarcopenia has been defined as low skeletal muscle mass (appendicular lean mass in age-related primary sarcopenia) and function, but 
muscle changes should be considered in the context of obesity.

Skeletal muscle mass and relative muscle mass: in absolute terms, high body fat in obesity may result in a relative reduction of skeletal muscle mass (\% skeletal muscle mass/BW), also in the absence of absolute skeletal muscle loss. A relative reduction in skeletal muscle mass could therefore merely result from higher body fat. Individuals with obesity may conversely have comparable or even higher absolute skeletal muscle mass relative to nonobese counterparts, due to higher overall body mass and potentially higher related muscle workload in daily physical activity $[80,81]$. However, near-normal skeletal muscle mass according to reference ranges for the general non-obese population may be accompanied by lower or inadequate muscle strength and performance in individuals with obesity [82].

For the definition of sarcopenia in SO we therefore support the concept of normalizing skeletal muscle mass to body mass and the need to introduce the concept of "relative or adequate muscle mass."

Skeletal muscle mass assessment: ideally, total skeletal muscle mass should be directly measured using gold standard techniques for the diagnosis of SO. This group, however, acknowledges that surrogates of skeletal muscle mass often need to be considered, due to practical limitations in its assessment in clinical and research settings. Even accurate methods commonly used in clinical research and practice provide assessment of lean mass (LM) or fat-free mass (FFM) as a whole, which includes other non-muscle organs such as gut, liver, kidney. Body fluids are also included in FFM and may reduce precision. Specific muscle areas can also be considered as surrogates and have been reported to predict clinical outcomes in specific disease settings [83, 84].

Skeletal muscle quality: among other specific issues, skeletal muscle quality may be profoundly altered in people with obesity, particularly in terms of ectopic fat deposition (e.g., myosteatosis) which may be highly prevalent in the presence of excess body fat. Myosteatosis is indeed recognized to negatively correlate with skeletal muscle mass and strength, as well as with systemic metabolic derangements including insulin resistance and type 2 diabetes, and with mobility, thereby being of prognostic relevance [85-87].

Skeletal muscle function: The group supports that sarcopenia in SO must be defined as concomitant reduction of skeletal muscle mass and function. Skeletal muscle functional parameters (primarily based on muscle strength) therefore need to be considered in the defini- tion of SO. Besides the relevance of muscle functional parameters for patient outcomes and prognosis, differential changes in skeletal muscle mass and functional parameters reported in several available studies directly support their independent measurement $[88,89]$.

Discrepancies in muscle mass and function may also be due, at least in part, to current methodological limitations in accurate assessment of skeletal muscle mass [86]. However, other relevant pathophysiological features including muscle quality and composition, muscle energy metabolism derangements and neuromuscular junction alterations may alter the relationship between skeletal muscle mass and function, thereby supporting the need to directly assess the latter to define and diagnose SO. Although skeletal muscle mass per se may be relevant for metabolic outcomes, altered muscle function is indeed associated with global individual functional impairment, reduced quality of life and shorter survival $[90,91]$.

Excess body fat may also notably lead per se to functional impairment and disability due to motor or cardiorespiratory complications. This also indicates the synergistic negative impact of both fat and skeletal muscle components on SO clinical outcomes.

Sarcopenic obesity epidemiology: SO is more frequently present in older adults, in part, due to the changes observed in body composition which in general accompany the aging process [65].

However, SO is not uniquely a geriatric condition (or limited to older adults). Several risk factors can accelerate the onset of sarcopenia in individuals with obesity such as clustered systemic and muscle oxidative stress, inflammation and insulin resistance which may occur in obesity particularly in the presence of metabolic complications and other comorbidities. Sarcopenic obesity can, therefore, also be observed in middle-aged and younger individuals with obesity often particularly due to acute and chronic diseases, and to rapid increments or body weight cycling also following weight loss treatment. In 28 of the 75 studies selected in our recent SO systematic review [20], the mean age of the studied population was below 65 years. In a study conducted by Poggiogalle et al. [75] in a sample of 727 participants with obesity $(141 \mathrm{M}, 586$ F; mean age: $45.6 \pm 13.5$ and $45.8 \pm 13.6$ years; mean BMI: $37.6 \pm 6$ and $37.8 \pm 5.8 \mathrm{~kg} / \mathrm{m}^{2}$, respectively), the prevalence of SO (sarcopenia defined as ALM/W $<2$ SD than the sex-specific mean reference value of a young population) was $34.8 \%$ in males and $50.1 \%$ in females. Similar results were obtained by El Ghoch et al. [92] in a sample of 147 individuals with obesity, where $63.3 \%$ met the cri- 
teria for reduced lean mass that was negatively associated with the 6-min walking test.

Sarcopenic obesity classification - primary and secondary sarcopenic obesity: SO, and in particular its sarcopenia component, has been proposed to be differentiated into a primary or secondary condition, with primary sarcopenia defined as age-related, or linked to sedentary lifestyle or to the direct effect of adipose tissueassociated inflammation on muscle mass. On the other hand, secondary sarcopenia is considered to result from the presence of obesity itself as an accelerating factor, and acute diseases which may provide the major pathophysiological background for the condition, with vicious cycling of muscle catabolic derangements, low physical activity, and fat gain. In the present consensus, no differentiation was proposed between primary and secondary sarcopenia in terms of definition and diagnostic criteria due to lack of evidence for differential defining features. This classification may be however useful to better understand the aetiopathogenetic mechanisms leading to $\mathrm{SO}$, and the proposed staging phase following SO diagnosis may provide clues regarding the presence of a primary or secondary form of SO. Notably, future research should also aim at differentiating the potential two SO forms, particularly since differential treatment strategies may be needed to address differential prevalent underlying mechanisms.

\section{Sarcopenic Obesity Diagnostic Procedures}

a. Screening

Screening tools should have maximum sensitivity. The ability to screen needs to be practical, affordable and time efficient for all at-risk individuals and potentially performed by different health care professionals (HCPs; e.g., nurses, general practitioners) who may or may not have expertise in the field of obesity or sarcopenia). It should therefore be carried out with tools that are easy to apply in a variety of clinical settings (e.g., outpatient clinics, hospitals, skilled nursing homes). It is important to note that the choice of reference ranges for each race or ethnic group has been challenging to identify given the lack of this information in some studies and mixed ethnicities in others. The utmost caution must therefore be applied in the choice of references in clinical and research activities since most of the studies are in Caucasian groups.

The group supports that screening aims at case finding with maximized sensitivity, which should be accessible to the highest possible number of individuals at risk and should therefore be feasible to the highest number of HCP also without specialized expertise in the field of obe- sity or sarcopenia. For this reason, the group supports the use of accessible, affordable and easy to use tools. The use of BMI is considered to be fully acceptable in the screening phase of the diagnostic process, both for its wide utilization in routine clinical practice in all settings and to allow implementation of the screening procedure, while recognizing the important limitations of BMI in the detection of fat distribution and body composition $[24,25$, 93]. The group also supports the use of waist circumference $[27,28]$ as an accessible clinical tool that should be widely used for the identification of excess abdominal visceral fat and related cardiometabolic risk. The use of waist circumference will improve the risk assessment of sarcopenia due to a high potential catabolic impact of visceral fat-related abnormalities.

The group supports the list of conditions that raise clinical suspicion and risk factors including older age (Table 1). It is therefore proposed that all people with obesity and overweight above the age of 70 should be considered at risk of SO, due to the additional risk of developing age-related sarcopenia. In practice, it is proposed that this population should be regularly screened guided by muscle functional tests to rule out SO. Additional risk factors include several chronic diseases well known to enhance the risk of loss of muscle mass and function, as well as catabolic events or nutritional derangements potentially causing muscle loss [94, 95].

Major diseases that can affect body weight through fluid retention (e.g., heart, liver and kidney failure, cancer) should also be carefully considered and assessed to avoid screening errors. The group finally advocates for the development of questionnaires that should be validated to establish the risk of sarcopenia in individuals with obesity at all ages. For example, the SARC-F [31, 32] includes five components: strength, assistance walking, rise from a chair, climbing stairs and history of falls, and can be used in the screening phase for older adults, since it has not been currently validated in younger age categories. Studies have suggested that the sensitivity of SARCF may be improved by adding calf circumference [96]. Nonetheless, very limited data are however currently available on the use of calf circumference in SO, mainly reflecting the necessity to standardize the procedure [97] and the general limitations to the use of anthropometry in individuals with obesity, as only recently adjustment factors have been made available [98]. Future research will be needed to verify the potential usefulness of including calf circumference in the SO screening procedure [43, 98-103]. 


\section{b. Diagnosis}

Diagnosis of SO, following positive screening, requires the presence of both altered skeletal muscle functional parameters and altered body composition. Skeletal muscle mass, estimated using techniques available for clinical practice such as BIA and DXA that do not directly assess muscle, is not a good indicator of functional impairment [104]. In fact, when using the D3-creatine dilution method, the association between muscle mass and disability is significant [105].

Altered skeletal muscle functional parameters: As discussed above, the group agrees that muscle function is a fundamental component of SO in both the definition and diagnostic process. In particular, the group agrees that functional consequences are of the utmost importance in the definition of the clinical picture of SO since they can affect quality of life of patients and treatment protocols of SO. Not only in sarcopenia, but also in obesity, functional impairment is very frequent and may occur well before old age [106-108]. SO diagnosis, therefore, needs to include direct assessment of altered skeletal muscle functional parameters along with altered body composition.

The decision to recommend the assessment of skeletal muscle functional parameters as the first step in the diagnostic process also addresses the need for practicality. The evaluation of body composition requires tools that, although increasingly available, may be less routinely accessible than those employed for the assessment of skeletal muscle function, particularly regarding muscle strength and physical performance.

In this context, a preliminary evaluation of functional parameters may represent a feasible approach to assess the risk of SO in at-risk subjects with a positive screening but without functional impairment who therefore would not meet the diagnostic criteria for SO. On the other hand, impairment of skeletal muscle functional parameters mandates the continuation of the diagnostic process with assessment of body composition [109].

The group indicates that muscle strength should be the functional parameter of choice for SO diagnosis although the available data do not show, at the moment, a clear superiority of a specific functional test; as such, this is an important topic for future investigation. More generally, as shown in online supplementary Table S2 also for the chair stand test and knee extensor strength, reference values have been commonly generated based on studies characterized by great heterogeneity of the considered population (age, ethnicity, sex). While the definition or refinement of reference values represents a topic for future research, researchers and clinicians should currently refer to cut-off points that are the closest to their studied population or patients.

Although gait speed has clinical relevance as a measure of physical performance and may identify disability and functional impairment in various settings, the group does not recommend its inclusion as a primary mandatory assessment tool in the SO diagnostic procedure because of potential clinical confounding factors that may affect test results, including osteoarthritis of the knee which is frequently observed in patients with obesity [110].

While supporting utilization of muscle strength tests (hand grip strength and chair stand tests) that are not, or are less impacted by pain, the group advocates further research that could lead to a larger panel of tests for muscle function assessment in the SO diagnostic procedure. For practical purposes, several skeletal muscle strength assessment methods have been proposed in the literature, each with advantages and limitations including validation studies in specific age groups or in samples of patients with well-defined clinical pictures [111].

The group recognizes that available information does not allow to select a single optimal measurement system; different systems for evaluating skeletal muscle strength are therefore presented in online supplementary Table S2, and it is recommended to use validated cut-off points for different sex and age classes. The concept of relative muscle strength is potentially relevant, but the group agrees that there is currently no sufficient evidence to recommend adjustment methods based on body weight or $\mathrm{BMI}$, also considering that no clear cut-offs for relative HGS are available (with only the Foundation for the National Institutes of Health - FNIH - suggesting a cut-off for HGS/BMI) and that advantages and disadvantages of using normalized strength are still not clearly defined [33, 40, 112-115].

The group therefore supports the use of maximal strength between the two limbs for the definition of HGS and knee extensor strength. We encourage studies that further define optimal potential adjustment criteria, particularly for people with obesity.

Body composition assessment methods: With regard to body composition assessment, obesity should be defined as increased FM\% of total body weight. An increase in visceral FM should also be considered for the evaluation of overall clinical risk by measuring waist circumference [30]. Using normalizations for FM (e.g., fat mass index) may lead to different and partly contradictory results [116]. The panel supports normalization for body weight but is aware of the limits of this option (e.g., in case of significant increase in body water) and therefore advo- 
cates that further studies are needed to potentially validate the use of different adjustments of FM (e.g., for body height) in individuals with obesity in the process of SO diagnosis.

Moreover, the diagnosis of sarcopenia requires a relative reduction of skeletal muscle mass adjusted to body weight; the panel considers ALM/W as the most suitable parameter when DXA is available; ALM/W or SMM/W may represent a valid option for BIA. However, it should be noted that skeletal muscle mass is derived from equations when using BIA (developed from DXA), which may limit its reliability and requires caution in both clinical practice and research. In fact, the use of specific equations for BIA calculations should require validation methods that, together with cut-off points, may significantly differ from one study to another. Moreover, using BIA in individuals with $\mathrm{BMI}>34 \mathrm{~kg} / \mathrm{m}^{2}$ may lead to an underestimation of FM and an overestimation of FFM [117]. The proposal to identify sarcopenia in $\mathrm{SO}$ as a relative reduction of muscle mass adjusted to body weight is based on the observation that a relative reduction of muscle mass in the presence of high total body mass and FM may have relevant clinical and functional impact even in the absence of absolute muscle mass reduction [118].

In the systematic review that served as the background to this consensus process, the same number of papers used ALM/W or ALM/height ${ }^{2}$ as a parameter to define sarcopenia in SO. The panel advocates further studies to further verify the validity of each specific index, and particularly normalization for body height.

The group supports that body composition should be assessed by DXA, or BIA as second choice. Both methods have some acknowledged limitations. For DXA it includes cost issues, variability in tissue thickness, lean tissue hydration, lack of skeletal muscle quantification, the overall skeletal muscle proportion of lean mass and the inclusion of the non-muscle lean component that may reduce accuracy in persons with obesity and lead to discrepancies between body composition and functional parameters $[84,119,120]$. In turn, BIA limitations also include lack of direct measurement of body composition. Skeletal muscle mass is estimated based on BIA raw measurements based on population and device-specific equations, with reduced accuracy in the presence of altered fluid balance. The presence of obesity specially impacts the assumption of constant hydration potentially decreasing BIA accuracy [121, 122].

Also, age, ethnicity and specific disease conditions may require specific equations and cut-offs [82] that may not be available for different devices.

Definition and Diagnostic Criteria for Sarcopenic Obesity
In addition, as also discussed above, lack of direct measurement of skeletal muscle mass might lead to inaccuracies which could account, at least in part, for reported limited predictive value of DXA and BIA-estimated muscle mass with poor functional outcomes in older adults. In terms of methodology, use of DXA with visceral fat analyser does not appear to improve the definition of FM distribution, and its clinical applicability may be limited. The use of magnetic resonance imaging as well as D3creatine dilution is currently essentially limited to research settings; they may represent useful procedures to validate predictive equations and cut-off points to be used in clinical practice for the assessment of body composition and skeletal muscle mass.

Emerging research shows that the association of body composition, and particularly skeletal muscle mass, with functional parameters is stronger if tools directly measuring skeletal muscle mass such as D3-creatine dilution are employed [123]. However, D3-creatine dilution still awaits validation in various clinical settings and is not widely available for clinical practice implementation. While acknowledging that accurate direct measurement of skeletal muscle mass might improve its predictive value for muscle function, the group agrees that DXA and BIA represent an adequate compromise between precision and accuracy of measurements and availability of instruments for clinical implementation of SO diagnosis.

The group also emphasizes that CT has been increasingly employed in clinical research for measuring selected skeletal muscle areas, which have been validated against strong clinical outcomes in various disease settings. The third lumbar vertebra muscle landmark is recommended (from abdominal CT scans), based on its strong association with whole body muscle mass, although other muscles and muscle groups have been proposed, e.g. from chest, thigh scans and other areas. Notably, the use of CT scans specifically for routine diagnosis of sarcopenia is arguably difficult to implement, due to costs and X-ray exposure. Therefore, the use of this tool is largely limited to availability from medical records (e.g., opportunistic use) where CT scans are used in the evaluation of other disorders (e.g., malignancy). In this context, there is a strong rationale to incorporate body composition, which includes muscle mass assessment in large groups of patients undergoing CT examinations due to various disease conditions. Routine implementation of muscle assessment requires awareness of the clinical relevance of diagnosing low muscle mass and $\mathrm{SO}$ in the same patients.

Anthropometry, including calf circumference and mid-arm muscle circumference, may theoretically play a 
major role in the assessment of body composition in clinical practice by allowing for a simple and rapid estimation of skeletal muscle mass (e.g., markers of low muscle mass). Important limitations however apply for main anthropometric methods. Anthropometry is generally less sensitive than body composition methods described earlier. In addition, similar to other techniques, it only includes selected skeletal muscle groups. Most importantly, anthropometric measurements to estimate skeletal muscle mass are affected by excess subcutaneous fat or fluid accumulation such as commonly observed in obesity [124]. Therefore, use of anthropometry cannot be routinely recommended in people with obesity and should be limited to calf circumference and only in the absence of oedema. Recent data indicated that adjustment for BMI leads to a higher predictive validity of calf circumference as a marker of skeletal muscle mass and clinical outcomes in the Health and Nutrition Examination Survey (NHANES) general population cohort [98]. Further studies are urgently advocated to validate adjustment for BMI to detect low calf circumference, low skeletal muscle mass and potentially SO in persons with obesity [125].

Cut-offs: The group supports a general approach to use available consensus-based cut-offs at this time. Online supplementary Tables S1 and S2 provide a summary of main available examples of parameters considered in the present document. Age, sex and ethnicity-specific cut-offs are needed and may not be available for all devices and techniques in all clinical conditions.

With regard to skeletal muscle mass and to the "relative" muscle mass concept, the group advocates for the adjustment of skeletal muscle mass, and particularly ALM/W. Further research is needed to verify whether this adjustment leads to potential overestimation of muscle mass in people with obesity, whereas adjustment to height or BMI would better express the relative amount of muscle mass.

Regarding body FM, the upper sex-specific cut-offs of $40 \%$ for females and 30\% for males have been proposed as best predictors of mortality in the NHANES sample (American population) using DXA [59]. Woolcott et al. [126] developed a calculated \%FM parameter defined as relative FM based not on body composition assessment, but rather calculated using height and waist circumference. These values need to be validated in populations with different ethnicities and using different methods for $\% \mathrm{FM}$ assessment. While the group advocates normalization of SMM/W, the issue of normalization of FM by height is substantially less studied and may potentially lead to relevant differences in diagnosis and prevalence of SO. The group supports the use of \%FM while advocating comparative studies to validate these approaches and in particular normalization of \%FM for body height [116] against clinical outcomes and with one another.

\section{c. Staging}

The group supports SO staging after diagnosis has been established, based on the presence of complications attributable to altered body composition and skeletal muscle functional parameters such as metabolic diseases, functional impairments resulting from high FM and/or low muscle mass, cardiovascular, bowel and respiratory diseases. Although functional impairment mainly represents a consequence of $\mathrm{SO}$ with strong negative prognostic value, it may also be considered an aggravating or perpetuating factor as it may exert a negative independent impact on maintenance or recovery of an adequate body composition and function.

The possibility to consider global functional outcomes (e.g., quality of life related to mobility, institutionalization, disability) as markers of severity of SO and their inclusion in the staging of SO was also considered but not accepted since they may not be necessarily associated with (or only with) SO, also being more relevant for SO in older adults.

The proposed staging is aimed at stratifying patients based on clinical severity and higher risk for poor outcomes, and therefore in need for more aggressive treatment and follow-up. The group is aware that the proposal has not been investigated yet and is therefore entirely based on clinical expert opinion at this time. The group advocates for research on this topic as a relevant clinical priority and a future objective of SO-related studies.

\section{Limitations and Strengths}

The current statements and proposals are based on expert consensus from an international panel of researchers. A strength of the initiative is that the group is an international and multidisciplinary one, composed of representatives from four continents and different areas: clinical nutrition, obesity and sarcopenia, geriatrics with strong publication records in the field. The statements therefore represent a summary of wide and different perspectives and may enhance acceptance of the proposed algorithm. Notwithstanding, it is acknowledged as a potential limitation that the group was self-appointed, initiated by ESPEN and EASO representatives (L.M.D., L.B., S.B., T.C., R.B.) with subsequent selection of the expert panel. 
Lack of consistency in operational criteria to define $\mathrm{SO}$ and to select SO patients in previous clinical research is a general limitation that may affect any consensus initiative in the field. The current statements are therefore admittedly not necessarily evidence-based, but rather expert-based. Discussions were carried out at the highest level of rigorousness with the goal to account for all potential problems and limitations. The group also aimed at providing criteria that are suitable for wide implementation in clinical practice, with the goal to empower nonspecialist HCPs in the screening procedure, and to involve specialized professionals in the diagnostic and staging phases. There is a need to balance practicality (time, availability, cost) and an ideal scenario allowing maximum of precision with highest specificity and sensitivity. We acknowledge that there will likely be a need to differentiate assessments between clinical versus research settings. In the latter case, more sophisticated and precise methods should be used while in the clinical scenario availability of instruments will drive assessments of choice, which should anyway be based on the best applicable evidence. The current diagnostic algorithm is considered to be suitable for clinical practice at this stage, and the group plans to revise the proposal within a reasonable time frame of 3-5 years. New available data may then confirm the current framework or provide stronger alternatives. In particular, the group strongly encourages studies to address major areas of uncertainty, including but not limited to:

Cut-off values: as discussed, no universally accepted validated references are currently available for most of the parameters cited in this document. The present document provides data available in different settings (online suppl. Tables S1 and S2). In particular, there is an urgent need to establish whether parameters should be analysed using a $\mathrm{T}$ score (comparison with a young reference population) or referring to a coeval population (Z score). Finally, cut-offs should be validated as predictors of specific adverse outcomes (comorbidity, disability, mortality or other clinical outcomes).

Relative muscle and fat mass: the group supports the use of relative muscle mass, particularly appendicular lean mass whenever available normalized for body weight. In addition, the group recognizes the importance of the concept of relative FM, although its use in available research makes its implementation more difficult. The group highlights the need of rigorous comparative research to establish optimal utilization and normalization of absolute muscle and FM as established by body composition assessment.

Definition and Diagnostic Criteria for Sarcopenic Obesity
Muscle functional parameters in secondary sarcopenic obesity: the role of skeletal muscle function and primary assessment of muscle functional parameters has been discussed. The group acknowledges that including altered muscle functional parameters as a necessary component to complete the diagnostic process may lead to potential borderline conditions, particularly in younger patients with relative low muscle mass for their age but still relatively preserved muscle function. The group therefore advocates for studies to specifically analyse the predictive value of the proposed algorithm in detection of $\mathrm{SO}$ in younger subjects. The group is also aware that functional parameters have not been the primary outcome of interest in the vast majority of available studies on secondary sarcopenia or secondary SO in patients with cancer, other chronic conditions, or hospitalized e.g., in intensive care unit, where younger age may be more prevalent and body composition is likely to be more feasible and relevant than functional assessment. Future research is needed to establish the role of functional parameters on clinical outcomes in SO patients in this context.

\section{Conclusions}

ESPEN and EASO represented by the expert panel advocate that the proposed SO definition and diagnostic criteria be implemented in clinical practice and in interventional randomized controlled trials aimed in particular at exploring the impact of specific interventions on SO. In addition, they strongly encourage validation and prospective follow-up studies as well as secondary analysis of existing cohorts with the aim to increase the scientific evidence needed to identify and treat SO patients.

\section{Conflict of Interest Statement}

None of the authors display any conflict of interest in the production of this paper.

\section{Funding Sources}

This work did not receive any grant from funding agencies in the public, commercial or not-for-profit sectors. 


\section{Author Contributions}

Conceptualization: L.M. Donini, R. Barazzoni, L. Busetto, T. Cederholm, S. Bischoff; methodology: L.M. Donini, R. Barazzoni, L. Busetto, T. Cederholm, S. Bischoff; formal analysis: E. Parrinello; writing (original draft): L.M. Donini, R. Barazzoni, L. Busetto, T. Cederholm, S. Bischoff; writing (review and editing): all the authors; visualization: L.M. Donini, R. Barazzoni, L. Busetto, T. Cederholm, S. Bischoff; supervision: all the authors.

\section{Data Availability Statement}

All data generated or analysed during this study are included in this article and in the supplementary material mentioned above. Further enquiries can be directed to the corresponding author.

\section{References}

1 Barazzoni R, Bischoff SC, Boirie Y, Busetto L, Cederholm T, Dicker D, et al. Sarcopenic obesity: time to meet the challenge. Clin Nutr. 2018 Dec;37(6 6 Pt A):1787-93.

2 Barazzoni R, Bischoff S, Boirie Y, Busetto L, Cederholm T, Dicker D, et al. Sarcopenic obesity: time to meet the challenge. Obes Facts. 2018;11(4):294-305.

3 Cruz-Jentoft AJ, Landi F, Topinková E, Michel JP. Understanding sarcopenia as a geriatric syndrome. Curr Opin Clin Nutr Metab Care. 2010 Jan;13(1):1-7.

4 Makizako H. Frailty and Sarcopenia as a Geriatric Syndrome in Community-Dwelling Older Adults. Int J Environ Res Public Health. 2019 Oct;16(20):4013.

5 Cruz-Jentoft AJ, Bahat G, Bauer J, Boirie Y, Bruyère $\mathrm{O}$, Cederholm $\mathrm{T}$, et al.; Writing Group for the European Working Group on Sarcopenia in Older People 2 (EWGSOP2), and the Extended Group for EWGSOP2. Sarcopenia: revised European consensus on definition and diagnosis. Age Ageing. 2019 Jan;48(1):16-31.

6 Hong SH, Choi KM. Sarcopenic Obesity, Insulin Resistance, and Their Implications in Cardiovascular and Metabolic Consequences. Int J Mol Sci. 2020 Jan;21(2):494.

7 Prokopidis K, Cervo MM, Gandham A, Scott D. Impact of Protein Intake in Older Adults with Sarcopenia and Obesity: A Gut Microbiota Perspective. Nutrients. 2020 Jul;12(8):2285.

8 Pekař $\mathrm{M}$, Pekařová $\mathrm{A}$, Bužga $\mathrm{M}$, Holéczy $\mathrm{P}$, Soltes M. The risk of sarcopenia 24 months after bariatric surgery - assessment by dual energy X-ray absorptiometry (DEXA): a prospective study. Wideochir Inne Tech Malo Inwazyjne. 2020 Dec;15(4):583-7.

9 Wang M, Tan Y, Shi Y, Wang X, Liao Z, Wei P. Diabetes and sarcopenic obesity: pathogenesis, diagnosis, and treatments. Front Endocrinol (Lausanne). 2020 Aug;11:568.

10 Sherf-Dagan S, Zelber-Sagi S, Buch A, Bar N, Webb M, Sakran N, et al. Prospective Longitudinal Trends in Body Composition and Clinical Outcomes 3 Years Following Sleeve Gastrectomy. Obes Surg. 2019 Dec;29(12):3833-41.
11 Rossi AP, Rubele S, Calugi S, Caliari C, Pedelini F, Soave F, et al. Weight Cycling as a Risk Factor for Low Muscle Mass and Strength in a Population of Males and Females with Obesity. Obesity (Silver Spring). 2019 Jul;27(7):1068-75.

12 Guillet C, Masgrau A, Walrand S, Boirie Y. Impaired protein metabolism: interlinks between obesity, insulin resistance and inflammation. Obes Rev. 2012;13(Suppl 2):51e7.

13 Baumgartner RN. Body composition in healthy aging. Ann N Y Acad Sci. 2000 May;904(1):437-48.

14 Flegal KM, Carroll MD, Ogden CL, Johnson CL. Prevalence and trends in obesity among US adults, 1999-2000. JAMA 2002;288(14):1723e7.

15 Villareal DT. Obesity in older adults - a growing problem. In: Watkins Bales C, Seel Ritchie $\mathrm{C}$, editors. Nutrition and health: handbook of clinical nutrition and aging. 2nd ed. New York: Humana Press; 2009. p. $263 \mathrm{e} 77$.

16 Bauer JM, Cruz-Jentoft AJ, Fielding RA, Kanis JA, Reginster JY, Bruyère $\mathrm{O}$, et al. Is there enough evidence for osteosarcopenic obesity as a distinct entity? A critical literature review.CalcifTissueInt.2019Aug;105(2):10924.

17 Peng TC, Chen WL, Chen YY, Chao YP, Wu LW, Kao TW. Associations between different measurements of sarcopenic obesity and health outcomes among non-frail community-dwelling older adults in Taiwan. Br J Nutr. 2021 Dec;126(11):1749-57.

18 Atkins JL, Wannamathee SG. Sarcopenic obesity in ageing: cardiovascular outcomes and mortality. Br J Nutr. 2020 Nov;124(10):110213.

19 Du Y, Wang X, Xie H, Zheng S, Wu X, Zhu X, et al. Sex differences in the prevalence and adverse outcomes of sarcopenia and sarcopenic obesity in community dwelling elderly in East China using the AWGS criteria. BMC Endocr Disord. 2019 Oct;19(1):109.

20 Donini LM, Busetto L, Bauer JM, Bischoff S, Boirie Y, Cederholm T, et al. Critical appraisal of definitions and diagnostic criteria for sarcopenic obesity based on a systematic review. Clin Nutr. 2020 Aug;39(8):2368-88.
21 Bischoff SC, Singer P, Koller M, Barazzoni R, Cederholm T, van Gossum A. Standard operating procedures for ESPEN guidelines and consensus papers. Clin Nutr. 2015 Dec;34(6):1043-51.

22 WHO. Obesity: preventing and managing the global epidemic. Report on a WHO consultation on obesity, Geneva, 3-5 June, 1997. WHO/NUT/NCD/98.1. Technical Report Series Number 894. Geneva: World Health Organization; 2000.

23 WHO Expert Consultation. Appropriate body-mass index for Asian populations and its implications for policy and intervention strategies. Lancet. 2004 Jan;363(9403):15763.

24 Shah NR, Braverman ER. Measuring adiposity in patients: the utility of body mass index (BMI), percent body fat, and leptin. PLoS One. 2012;7(4):e33308

25 Batsis JA, Mackenzie TA, Bartels SJ, Sahakyan KR, Somers VK, Lopez-Jimenez F. Diagnostic accuracy of body mass index to identify obesity in older adults: NHANES 1999-2004. Int J Obes. 2016 May;40(5):761-7.

26 Examination Committee of Criteria for Obesity Disease in Japan; Japan Society for the Study of Obesity. New criteria for "obesity disease." Jpn Circ J. 2002 Nov;66(11):987-92.

27 Lean ME, Han TS, Morrison CE. Waist circumference as a measure for indicating need for weight management. BMJ. 1995 Jul;311(6998):158-61.

28 National Institutes of Health. Clinical Guidelines on the Identification, Evaluation, and Treatment of Overweight and Obesity in Adults - The Evidence Report. Obes Res. 1998 Sep;6 Suppl 2:51S-209S.

29 Misra A, Vikram NK, Gupta R, Pandey RM, Wasir JS, Gupta VP. Waist circumference cutoff points and action levels for Asian Indians for identification of abdominal obesity. Int J Obes. 2006 Jan;30(1):106-11.

30 Wang Z, Ma J, Si D. Optimal cut-off values and population means of waist circumference in different populations. Nutr Res Rev. 2010 Dec;23(2):191-9.

31 Malmstrom TK, Morley JE. SARC-F: a simple questionnaire to rapidly diagnose sarcopenia. J Am Med Dir Assoc. 2013 Aug;14(8):531-2. 
32 Woo J, Leung J, Morley JE. Validating the SARC-F: a suitable community screening tool for sarcopenia? J Am Med Dir Assoc. 2014 Sep;15(9):630-4.

33 Ha YC, Yoo JI, Park YJ, Lee CH, Park KS. Measurement of Uncertainty Using Standardized Protocol of Hand Grip Strength Measurement in Patients with Sarcopenia. J Bone Metab. 2018 Nov;25(4):243-9.

34 Kanada Y, Sakurai H, Sugiura Y, Arai T, Koyama S, Tanabe S. Estimation of 1RM for knee extension based on the maximal isometric muscle strength and body composition. J Phys Ther Sci. 2017 Nov;29(11):2013-7.

35 Guralnik JM, Simonsick EM, Ferrucci L, Glynn RJ, Berkman LF, Blazer DG, et al. A short physical performance battery assessing lower extremity function: association with self-reported disability and prediction of mortality and nursing home admission. J Gerontol. 1994 Mar;49(2):M85-94.

36 Jones CJ, Rikli RE, Beam WC. A 30-s chairstand test as a measure of lower body strength in community-residing older adults. Res Q Exerc Sport. 1999 Jun;70(2):113-9.

37 Orwig DL, Magaziner J, Fielding RA, Zhu H, Binder EF, Cawthon PM, et al. Application of SDOC Cut Points for Low Muscle Strength for Recovery of Walking Speed After Hip Fracture. J Gerontol A Biol Sci Med Sci. 2020 Jun;75(7):1379-85.

38 Dodds RM, Syddall HE, Cooper R, Benzeval M, Deary IJ, Dennison EM, et al. Grip strength across the life course: normative data from twelve British studies. PLoS One. 2014 Dec;9(12):e113637.

39 Bhasin S, Travison TG, Manini TM, Patel S, Pencina KM, Fielding RA, et al. Sarcopenia Definition: The Position Statements of the Sarcopenia Definition and Outcomes Consortium. J Am Geriatr Soc. 2020 Jul;68(7):1410-8.

40 Patel SM, Duchowny KA, Kiel DP, Correade-Araujo R, Fielding RA, Travison T, et al. Sarcopenia Definition \& Outcomes Consortium Defined Low Grip Strength in Two Cross-Sectional, Population-Based Cohorts. I Am Geriatr Soc. 2020 Jul;68(7):1438-44

41 Lauretani F, Russo CR, Bandinelli S, Bartali B, Cavazzini C, Di Iorio A, et al. Age-associated changes in skeletal muscles and their effect on mobility: an operational diagnosis of sarcopenia. J Appl Physiol (1985). 2003 Nov;95(5):1851-60.

42 Studenski SA, Peters KW, Alley DE, Cawthon PM, McLean RR, Harris TB, et al. The FNIH sarcopenia project: rationale, study description, conference recommendations, and final estimates. J Gerontol A Biol Sci Med Sci. 2014 May;69(5):547-58.

43 Chen LK, Woo J, Assantachai P, Auyeung TW, Chou MY, Iijima K, et al. Asian Working Group for Sarcopenia: 2019 Consensus Update on Sarcopenia Diagnosis and Treatment. J Am Med Dir Assoc. 2020 Mar;21(3):300307.e2.
44 Auyeung TW, Arai H, Chen LK, Woo J. Letter to the editor: normative data of handgrip strength in 26,344 older adults - a pooled dataset from eight cohorts in Asia. J Nutr Health Aging. 2020;24(1):125-6.

45 Spruit MA, Sillen MJ, Groenen MT, Wouters EF, Franssen FM. New normative values for handgrip strength: results from the UK Biobank. J Am Med Dir Assoc. 2013 Oct;14(10):775.e5-11.

46 Assantachai $\mathrm{P}$, Muangpaisan W, Intalapaporn S, Sitthichai K, Udompunturak S. Cutoff points of quadriceps strength, declines and relationships of sarcopenia-related variables among Thai community-dwelling older adults. Geriatr Gerontol Int. 2014 Feb;14 Suppl 1:61-8.

47 Martien S, Delecluse C, Boen F, Seghers J, Pelssers J, Van Hoecke AS, et al. Is knee extension strength a better predictor of functional performance than handgrip strength among older adults in three different settings? Arch Gerontol Geriatr. 2015 Mar-Apr;60(2):252-8.

48 Cesari M, Kritchevsky SB, Newman AB, Simonsick EM, Harris TB, Penninx BW, et al.; Health, Aging and Body Composition Study. Added value of physical performance measures in predicting adverse health-related events: results from the Health, Aging And Body Composition Study. J Am Geriatr Soc. $2009 \mathrm{Feb} ; 57(2): 251-9$.

49 Rikli RE, Jones CJ. Development and validation of criterion-referenced clinically relevant fitness standards for maintaining physical independence in later years. Gerontologist. 2013 Apr;53(2):255-67.

50 Gallagher D, Heymsfield SB, Heo M, Jebb SA, Murgatroyd PR, Sakamoto Y. Healthy percentage body fat ranges: an approach for developing guidelines based on body mass index. Am J Clin Nutr. 2000 Sep;72(3):694-701.

51 Baumgartner RN, Koehler KM, Gallagher D, Romero L, Heymsfield SB, Ross RR, et al. Epidemiology of sarcopenia among the elderly in New Mexico. Am J Epidemiol. 1998 Apr;147(8):755-63.

52 Ishii S, Chang C, Tanaka T, Kuroda A, Tsuji $\mathrm{T}$, Akishita M, et al. The Association between Sarcopenic Obesity and Depressive Symptoms in Older Japanese Adults. PLoS One. 2016 Sep;11(9):e0162898.

53 Bahat G, Kilic C, Topcu Y, Aydin K, Karan MA. Fat percentage cutoff values to define obesity and prevalence of sarcopenic obesity in community-dwelling older adults in Turkey. Aging Male. 2020 Dec;23(5):477-82.

54 Zoico E, Di Francesco V, Guralnik JM, Mazzali G, Bortolani A, Guariento S, et al. Physical disability and muscular strength in relation to obesity and different body composition indexes in a sample of healthy elderly women. Int J Obes. 2004 Feb;28(2):234-41.
55 Pedrero-Chamizo R, Gómez-Cabello A, Meléndez A, Vila-Maldonado S, Espino L, Gusi N, et al. Higher levels of physical fitness are associated with a reduced risk of suffering sarcopenic obesity and better perceived health among the elderly: the EXERNET multi-center study. J Nutr Health Aging. 2015 Feb;19(2):211-7.

56 Kim TN, Yang SJ, Yoo HJ, Lim KI, Kang HJ, Song W, et al. Prevalence of sarcopenia and sarcopenic obesity in Korean adults: the Korean sarcopenic obesity study. Int J Obes. 2009 Aug;33(8):885-92.

57 Lee J, Hong YP, Shin HJ, Lee W. Associations of Sarcopenia and Sarcopenic Obesity With Metabolic Syndrome Considering Both Muscle Mass and Muscle Strength. J Prev Med Public Health. 2016 Jan;49(1):35-44.

58 Seger JC, Horn DB, Westman EC, Lindquist R, Scinta W, Richardson LA, et al. American Society of Bariatric Physicians Obesity Algorithm: Adult Adiposity Evaluation and Treatment 2013 [cited 2021 Oct 20]. Available from: www.obesityalgorithm.org.

59 Woolcott OO, Bergman RN. Defining cutoffs to diagnose obesity using the relative fat mass (RFM): association with mortality in NHANES 1999-2014. Int J Obes. 2020 Jun;44(6):1301-10.

60 Davison KK, Ford ES, Cogswell ME, Dietz $\mathrm{WH}$. Percentage of body fat and body mass index are associated with mobility limitations in people aged 70 and older from NHANES III. J Am Geriatr Soc. 2002 Nov;50(11):18029.

61 Janssen I, Heymsfield SB, Ross R. Low relative skeletal muscle mass (sarcopenia) in older persons is associated with functional impairment and physical disability. J Am Geriatr Soc. 2002 May;50(5):889-96.

62 Lim S, Kim JH, Yoon JW, Kang SM, Choi SH, Park YJ, et al. Sarcopenic obesity: prevalence and association with metabolic syndrome in the Korean Longitudinal Study on Health and Aging (KLoSHA). Diabetes Care. 2010 Jul;33(7):1652-4.

63 An KO, Kim J. Association of Sarcopenia and Obesity With Multimorbidity in Korean Adults: A Nationwide Cross-Sectional Study. J Am Med Dir Assoc. 2016 Oct;17(10):960. e1-7.

64 Baek SJ, Nam GE, Han KD, Choi SW, Jung SW, Bok AR, et al. Sarcopenia and sarcopenic obesity and their association with dyslipidemia in Korean elderly men: the 2008-2010 Korea National Health and Nutrition Examination Survey. J Endocrinol Invest 2014;37(3):247e60.

65 Batsis JA, Barre LK, Mackenzie TA, Pratt SI, Lopez-Jimenez F, Bartels SJ. Variation in the prevalence of sarcopenia and sarcopenic obesity in older adults associated with different research definitions: dual-energy $\mathrm{X}$-ray absorptiometry data from the National Health and Nutrition Examination Survey 19992004. J Am Geriatr Soc 2013;61(6):974e80. 
66 Cho Y, Shin SY, Shin MJ. Sarcopenic obesity is associated with lower indicators of psychological health and quality of life in Koreans. Nutr Res. 2015;35(5):384e92.

67 Chung JY, Kang HT, Lee DC, Lee HR, Lee YJ. Body composition and its association with cardiometabolic risk factors in the elderly: a focus on sarcopenic obesity. Arch Gerontol Geriatr. 2013 Jan-Feb;56(1):270-8.

68 Hwang B, Lim JY, Lee J, Choi NK, Ahn YO, Park BJ. Prevalence rate and associated factors of sarcopenic obesity in Korean elderly population. J Korean Med Sci. 2012;27(7): $748 \mathrm{e} 55$.

69 Kim JH, Cho JJ, Park YS. Relationship between sarcopenic obesity and cardiovascular disease risk as estimated by the Framingham risk score. J Korean Med Sci. 2015 Mar;30(3):264-71.

70 Kim YS, Lee Y, Chung YS, Lee DJ, Joo NS, Hong D, et al. Prevalence of sarcopenia and sarcopenic obesity in the Korean population based on the fourth Korean national health and nutritional examination surveys. J Gerontol A Biol Sci Med Sci 2012;67(10):1107e13.

71 Kim MK, Baek KH, Song KH, Il Kang M, Park $\mathrm{CY}$, Lee WY, et al. Vitamin D deficiency is associated with sarcopenia in older Koreans, regardless of obesity: the Fourth Korea National Health and Nutrition Examination Surveys (KNHANES IV) 2009. J Clin Endocrinol Metab. 2011 Oct;96(10):3250-6.

72 Lee S, Kim TN, Kim SH. Sarcopenic obesity is more closely associated with knee osteoarthritis than is nonsarcopenic obesity: a crosssectional study. Arthritis Rheum. 2012;64(12): 3947e54.

73 Lee YH, Jung KS, Kim SU, Yoon HJ, Yun YJ, Lee BW, et al. Sarcopaenia is associated with NAFLD independently of obesity and insulin resistance: nationwide surveys (KNHANES 2008-2011). J Hepatol 2015;63(2):486e93.

74 Oh C, Jho S, No JK, Kim HS. Body composition changes were related to nutrient intakes in elderly men but elderly women had a higher prevalence of sarcopenic obesity in a population of Korean adults. Nutr Res 2015;35 (1):1e6.

75 Poggiogalle E, Lubrano C, Sergi G, Coin A, Gnessi L, Mariani S, et al. Sarcopenic obesity and metabolic syndrome in adult Caucasian subjects. J Nutr Health Aging 2016;20(9): $958 \mathrm{e} 63$.

76 Chun SW, Kim W, Choi KH. Comparison between grip strength and grip strength divided by body weight in their relationship with metabolic syndrome and quality of life in the elderly. PLoS One. 2019 Sep;14(9):e0222040.

77 Chon D, Shin J, Kim JH. Consideration of body mass index (BMI) in the association between hand grip strength and hypertension: Korean Longitudinal Study of Ageing (KLoSA). PLoS One. 2020 Oct;15(10): e0241360.
78 Batsis JA, Villareal DT. Sarcopenic obesity in older adults: aetiology, epidemiology and treatment strategies. Nat Rev Endocrinol. 2018 Sep;14(9):513-37.

79 Koliaki C, Liatis S, Dalamaga M, Kokkinos A. Sarcopenic Obesity: Epidemiologic Evidence, Pathophysiology, and Therapeutic Perspectives. Curr Obes Rep. 2019 Dec;8(4):458-71.

80 Tomlinson DJ, Erskine RM, Morse CI, Winwood K, Onambélé-Pearson G. The impact of obesity on skeletal muscle strength and structure through adolescence to old age. Biogerontology. 2016 Jun;17(3):467-83.

81 Villareal DT, Banks M, Siener C, Sinacore DR, Klein S. Physical frailty and body composition in obese elderly men and women. Obes Res. 2004 Jun;12(6):913-20.

82 Walowski CO, Braun W, Maisch MJ, Jensen B, Peine S, Norman K, et al. Reference Values for Skeletal Muscle Mass - Current Concepts and Methodological Considerations. Nutrients. 2020 Mar;12(3):755.

83 MacDonald AJ, Greig CA, Baracos V. The advantages and limitations of cross-sectional body composition analysis. Curr Opin Support Palliat Care. 2011 Dec;5(4):342-9.

84 Prado CM, Heymsfield SB. Lean tissue imaging: a new era for nutritional assessment and intervention. JPEN J Parenter Enteral Nutr. 2014 Nov;38(8):940-53.

85 Correa-de-Araujo R, Addison O, Miljkovic I, Goodpaster BH, Bergman BC, Clark RV, et al. Myosteatosis in the context of skeletal muscle function deficit: an interdisciplinary workshop at the National Institute on Aging. Front Physiol. 2020 Aug;11:963.

86 Tardif N, Salles J, Guillet C, Tordjman J, Reggio S, Landrier JF, et al. Muscle ectopic fat deposition contributes to anabolic resistance in obese sarcopenic old rats through eIF2 $\alpha$ activation. Aging Cell. 2014 Dec;13(6):1001-11.

87 Kalinkovich A, Livshits G. Sarcopenic obesity or obese sarcopenia: a cross talk between ageassociated adipose tissue and skeletal muscle inflammation as a main mechanism of the pathogenesis. Ageing Res Rev. 2017 May;35:200-21.

88 Pahor M, Manini T, Cesari M. Sarcopenia: clinical evaluation, biological markers and other evaluation tools. J Nutr Health Aging. 2009 Oct;13(8):724-8.

89 Chang JS, Kim TH, Kim H, Choi EH, Kim N, Kong ID. Qualitative muscle mass index as a predictor of skeletal muscle function deficit in Asian older adults. Geriatr Gerontol Int. 2017 Jan;17(1):99-107.

90 Batsis JA, Mackenzie TA, Lopez-Jimenez F, Bartels SJ. Sarcopenia, sarcopenic obesity, and functional impairments in older adults: National Health and Nutrition Examination Surveys 1999-2004. Nutr Res. 2015 Dec;35(12):1031-9.

91 Cheung CL, Lee GK, Au PC, Li GH, Chan M, Li HL, et al. Systematic review and meta-analysis of lean mass and mortality: rationale and study description. Osteoporos Sarcopenia. 2021 Mar;7 Suppl 1:S3-12.
92 El Ghoch M, Rossi AP, Calugi S, Rubele S, Soave F, Zamboni M, et al. Physical performance measures in screening for reduced lean body mass in adult females with obesity. Nutr Metab Cardiovasc Dis. 2018 Sep;28 (9):917-21.

93 Müller MJ, Braun W, Enderle J, Bosy-Westphal A. Beyond BMI: Conceptual Issues Related to Overweight and Obese Patients. Obes Facts. 2016;9(3):193-205.

94 Dhillon RJ, Hasni S. Pathogenesis and Management of Sarcopenia. Clin Geriatr Med. 2017 Feb;33(1):17-26.

95 Cruz-Jentoft AJ, Kiesswetter E, Drey M, Sieber CC. Nutrition, frailty, and sarcopenia. Aging Clin Exp Res. 2017 Feb;29(1):43-8.

96 Yang M, Hu X, Xie L, Zhang L, Zhou J, Lin J, et al. Screening Sarcopenia in Community-Dwelling Older Adults: SARC-F vs SARC-F Combined With Calf Circumference (SARC-CalF). J Am Med Dir Assoc. 2018 Mar;19(3):277.e1-8.

97 Kawakami R, Miyachi M, Sawada SS, Torii $\mathrm{S}$, Midorikawa T, Tanisawa K, et al. Cut-offs for calf circumference as a screening tool for low muscle mass: WASEDA'S Health Study. Geriatr Gerontol Int. 2020 Oct;20(10):94350.

98 Gonzalez MC, Mehrnezhad A, Razaviarab N, Barbosa-Silva TG, Heymsfield SB. Calf circumference: cutoff values from the NHANES 1999-2006. Am J Clin Nutr. 2021 Jun;113(6):1679-87.

99 Santos LP, Gonzalez MC, Orlandi SP, Bielemann RM, Barbosa-Silva TG, Heymsfield SB; COCONUT Study Group. New Prediction Equations to Estimate Appendicular Skeletal Muscle Mass Using Calf Circumference: results From NHANES 1999-2006. JPEN J Parenter Enteral Nutr. 2019 Nov;43(8):998-1007.

100 Li R, Hu X, Tan L, Xie L, Zhang L, Zhou J, et al. Screening for Sarcopenia with a Self-Reported Cartoon Questionnaire: Combining SARC-F with Finger-Ring Test. J Nutr Health Aging. 2020;24(10):1100-6.

101 Mo YH, Zhong J, Dong X, Su YD, Deng WY, Yao XM, et al. Comparison of three case finding methods for sarcopenia in community-dwelling older individuals. J Am Med Dir Assoc. 2021 Apr;22(4):746-750.e1.

102 Kim M, Won CW. Sarcopenia in Korean Community-Dwelling Adults Aged 70 Years and Older: Application of Screening and Diagnostic Tools From the Asian Working Group for Sarcopenia 2019 Update. J Am Med Dir Assoc. 2020 Jun;21(6):752-8.

103 Lim WS, Lim JP, Chew J, Tan AW. Calf Circumference as a Case-Finding Tool for Sarcopenia: Influence of Obesity on Diagnostic Performance. J Am Med Dir Assoc. 2020 Sep;21(9):1359-61. 
104 Cawthon PM, Travison TG, Manini TM, Patel S, Pencina KM, Fielding RA, et al. Establishing the Link Between Lean Mass and Grip Strength Cut Points With Mobility Disability and Other Health Outcomes: Proceedings of the Sarcopenia Definition and Outcomes Consortium Conference. J Gerontol A Biol Sci Med Sci. 2020 Jun;75 (7):1317-23.

105 Cawthon PM, Blackwell T, Cummings SR, Orwoll ES, Duchowny KA, Kado DM, et al. Muscle Mass Assessed by the D3-Creatine Dilution Method and Incident Self-reported Disability and Mortality in a Prospective Observational Study of Community-Dwelling Older Men. J Gerontol A Biol Sci Med Sci. 2021 Jan;76(1):123-30.

106 Brunani A, Sirtori A, Capodaglio P, Donini LM, Buscemi S, Carbonelli MG, et al.; ICFOBESITY group. Disability assessment in an Italian cohort of patients with obesity using an International Classification of Functioning, Disability and Health (ICF)-derived questionnaire. Eur J Phys Rehabil Med. 2021 Aug;57(4):630-8.

107 Donini LM, Rosano A, Di Lazzaro L, Lubrano C, Carbonelli M, Pinto A, et al. Impact of Disability, Psychological Status, and Comorbidity on Health-Related Quality of Life Perceived by Subjects with Obesity. Obes Facts. 2020;13(2):191-200.

108 Donini LM, Merola G, Poggiogalle E, Lubrano C, Gnessi L, Mariani S, et al. Disability, Physical Inactivity, and Impaired HealthRelated Quality of Life Are Not Different in Metabolically Healthy vs. Unhealthy Obese Subjects. Nutrients. 2016 Nov;8(12):759.

109 Beaudart C, Rolland Y, Cruz-Jentoft AJ, Bauer JM, Sieber C, Cooper C, et al. Assessment of Muscle Function and Physical Performance in Daily Clinical Practice: a position paper endorsed by the European Society for Clinical and Economic Aspects of Osteoporosis, Osteoarthritis and Musculoskeletal Diseases (ESCEO). Calcif Tissue Int. 2019 Jul;105(1):1-14.
110 Wang T, He C. Pro-inflammatory cytokines: the link between obesity and osteoarthritis. Cytokine Growth Factor Rev. 2018 Dec;44:38-50.

111 Mijnarends DM, Meijers JM, Halfens RJ, ter Borg S, Luiking YC, Verlaan S, et al. Validity and reliability of tools to measure muscle mass, strength, and physical performance in community-dwelling older people: a systematic review. J Am Med Dir Assoc. 2013 Mar;14(3):170-8.

112 Sénéchal M, Dionne IJ, Brochu M. Dynapenic abdominal obesity and metabolic risk factors in adults 50 years of age and older. J Aging Health. 2012 Aug;24(5):812-26.

113 Yi DW, Khang AR, Lee HW, Son SM, Kang YH. Relative handgrip strength as a marker of metabolic syndrome: the Korea National Health and Nutrition Examination Survey (KNHANES) VI (2014-2015). Diabetes Metab Syndr Obes. 2018 May;11:227-40.

114 Shen C, Lu J, Xu Z, Xu Y, Yang Y. Association between handgrip strength and the risk of new-onset metabolic syndrome: a population-based cohort study. BMJ Open. 2020 Oct;10(10):e041384.

115 McGrath R. Comparing absolute handgrip strength and handgrip strength normalized to body weight in aging adults. Aging Clin Exp Res. 2019 Dec;31(12):1851-3.

116 Genton L, Mareschal J, Karsegard VL, Achamrah N, Delsoglio M, Pichard C, et al. An Increase in Fat Mass Index Predicts a Deterioration of Running Speed. Nutrients. 2019 Mar;11(3):701.

117 Becroft L, Ooi G, Forsyth A, King S, Tierney A. Validity of multi-frequency bioelectric impedance methods to measure body composition in obese patients: a systematic review. Int J Obes. 2019 Aug;43(8):1497-507.

118 Chen LK, Lee WJ, Peng LN, Liu LK, Arai H, Akishita M; Asian Working Group for Sarcopenia. Recent advances in sarcopenia research in Asia: update from the Asian working group for sarcopenia. JAMDA 2016; 17:767.ele7.
119 Schautz B, Later W, Heller M, Müller MJ Bosy-Westphal A. Total and regional relationship between lean and fat mass with increasing adiposity - impact for the diagnosis of sarcopenic obesity. Eur J Clin Nutr. 2012 Dec;66(12):1356-61.

120 Heymsfield SB, Gonzalez MC, Lu J, Jia G, Zheng J. Skeletal muscle mass and quality: evolution of modern measurement concepts in the context of sarcopenia. Proc Nutr Soc. 2015 Nov;74(4):355-66.

121 Sizoo D, de Heide LJ, Emous M, van Zutphen T, Navis G, van Beek AP. Measuring Muscle Mass and Strength in Obesity: a Review of Various Methods. Obes Surg. 2021 Jan;31(1):384-93.

122 Jensen B, Braun W, Geisler C, Both M, Klückmann K, Müller MJ, et al. Limitations of Fat-Free Mass for the Assessment of Muscle Mass in Obesity. Obes Facts. 2019; 12(3):307-15.

123 Zanker J, Patel S, Blackwell T, Duchowny K, Brennan-Olsen S, Cummings SR, et al.; Osteoporotic Fractures in Men (MrOS) Study Group. Walking Speed and Muscle Mass Estimated by the D3-Creatine Dilution Method Are Important Components of Sarcopenia Associated With Incident Mobility Disability in Older Men: A Classification and Regression Tree Analysis. J Am Med Dir Assoc. 2020 Dec;21(12):1997-2002.e1.

124 Vasileiou AM, Bull R, Kitou D, Alexiadou K, Garvie NJ, Coppack SW. Oedema in obesity; role of structural lymphatic abnormalities. Int J Obes. 2011 Sep;35(9):1247-50.

125 Ishida Y, Maeda K, Nonogaki T, Shimizu A, Yamanaka Y, Matsuyama R, et al. Impact of edema on length of calf circumference in older adults. Geriatr Gerontol Int. 2019 Oct;19(10):993-8.

126 Woolcott OO, Bergman RN. Relative fat mass (RFM) as a new estimator of wholebody fat percentage - a cross-sectional study in American adult individuals. Sci Rep. 2018 Jul;8(1):10980. 